\title{
Providing IPTV in the IMS network
}

\author{
Ladislav Kockovic ${ }^{1 *}$, Ladislav Szeiff ${ }^{2}$ \\ ${ }^{1,2}$ Slovak University of Technology in Bratislava, Faculty of Electrical Engineering and Information Technology, Institute of \\ Multimedia Information and Communication Technologies, lkovičova 3, 81219 Bratislava, Slovak republic
}

\begin{abstract}
Providing IPTV in the IMS network is increasingly a problem solved today. All households are gradually passing through innovation and slowly every equipment becomes the so-called intelligent or smart and can be connected to a private or public network where it can be monitored, configured, diagnosed and then repaired.. This same upgrading takes place in television, and its current output is in the form of IPTV (Internet Protocol Television) provided in the IP Multimedia Subsystem Network, a new generation network that provides both audio and video data. IMS has originated in 1999 for the 3 GPP standard in the 5th edition when switching from a $2 \mathrm{G}$ network to a $3 \mathrm{G}$ network. It has supported the older GSM and GPRS networks, but also a new UMTS network. In the sixth edition, it also has began to work on WLAN networks, more voice-oriented, or speech recognition services. This paper focuses on the provision of IPTV (Internet Protocol Television) in the IMS (IP Multimedia Subsystem) network. The aim of our paper is to design IPTV services in the IMS network, define the IMS architecture and the IMS network components such as HSS (Home Subscriber Server), various types of CSCF (Call State Control Function), describe the functionality and services provided by IMS and IPTV and, finally, compare the quality of the IPTV service provided by our network and by network of selected operator.
\end{abstract}

\section{Introduction}

Analogue television broadcasted via antenna receivers was replaced by digital broadcasting, various television broadcasting methods such as DVB-T digital broadcasting, DVB-S / S2 satellite broadcasting, DVB-C coaxial cable broadcasting are being developed.

IPTV, however, differs mainly by distributing audio / video content. In IPTV, the subscriber chooses what he wants to watch, and the operator sends the selection into the device. This means that only one transmission is transmitted to the subscriber and no more transmissions at the same time. This saves the bandwidth, improves the quality of the service, and the operator has more control over the entire network.

IPTV is primarily implemented in private networks. It provides a wider choice of traceable channels, adds the ability to set up its own IPTV service via the application server in agreement with the administrator or network operator, and thus satisfies a larger set of users.

Many people are confusing IPTV with Internet TV. The truth is, that while Internet TV works on a public Internet network, IPTV on a private network. The private network transfer is under greater operator control and it takes care of the better quality of the service. Internet TV has a predominantly low bandwidth, with a huge delay, a high operating load, and therefore a low QoS. Internet TV broadcasts everything in the direction of broadcasting. IPTV is watched only by users who have allocated rights to the network and the data is transmitted from the source to the target.
Using IMS, IPTV can be used with several other services. The more services the user uses on the network, the less services are charged, because the network for which the services are provided is unified, so telecommunication operators do not incur any additional costs for introducing a new service. Integration with IMS services is a step forward for telecommunication operators to maintain and attract customers. Operators are better able to use the bandwidth provided to customers because they can use the full bandwidth for multiple services. $[1,2]$

First part of the paper contains the brief introduction into investigating field. Next part is focused on IMS architecture followed by characteristic of IPTV architecture, which cosists of several basic elements, e. g. content sources, IP gateway, VoD server, etc. Fourth chapter deals with IPTV architecture in IMS environment, basic functions and elements are mentioned there. Fifth part contains design of an IPTV network architecture followed by comparision of quality of service in explored cases.

\section{IMS architecture}

The IMS architecture is quite complex, so we will divide the entire architecture into groups and subgroups. IMS consists of several parts that can be linked or distributed across the network. Architecture can be divided into several primary areas:

- User Interface Area:

* Corresponding author: ladislav.kockovic@gmail.com 
Part of the architecture placed on the user side - the endpoint.

- Access layer:

This is part of the architecture through which an overall network approach is implemented.

- Control layer:

Provides all IMS core features.

- Application layer:

Provides end-user access to a full-service web site and network controls. [3, 4]

\section{IPTV architecture}

The source signal is captured on the so-called operator's main distribution station, and then is resent to the local servers via the OSI transport layer. Next, it goes to a DSLAM (Digital Subscriber Line Access Multiplexer) DSLAM or Optical Line Termination (OLT), [5] depending on whether it is a digital subscriber line $\mathrm{xDSL}$ (x Digital Subscriber Line) or fiber optic connections, up to users. The user can choose anytime and any broadcast station. [6, 7]

\subsection{Content sources}

For example, terrestrial broadcasters transmitting DVB$\mathrm{T}$ signals, satellite or cable transmitters can be understood as content sources. The transmitted signal is transmitted to the receiving devices or antennas, the entire signal is sampled, encoded, and the multicast begins to send. [8]

\subsection{DVB - IP Gateway}

The gateway is decoding the incoming signal and transmitting it in the form of audio / video. Can handle over $100 \mathrm{TV}$ stations. It works as encoder, decoder, router and provides signal transmission. [8,9]

\subsection{Conditional access system}

It handles content encryption using a Common Scrambling Algorithm (CSA) encryption coming from a DVB-IP gateway or Video on Demand (VOD) server to ensure unprotected transmission. Only users with the appropriate key and pre-paid IPTV service can access the content. The system also allows tarification and control of users' services. The client decodes the stream using the set top box. [8]

\subsection{Server VoD / NVoD}

VoD, or NVoD (Near Video on Demand), provides the so-called video on demand from the program menu. It is in multiple locations in network architecture. It works with TV content or video content with MPEG-2 or MPEG-4 compression, each of which needs a bandwidth of approximately $4 \mathrm{Mbit} / \mathrm{s}$. It works in both multicast and unicast modes. The stored files have internal memory that reaches the value of ten terabytes. Users have access to these data through the GUI per the IP Set-Top Box and can choose from a wide range of recorded movies or recorded TV broadcasts. Using the same concept, it is possible to use image capture or broadcast services to Set-top box. $[7,8]$

\subsection{Billing system}

The billing system takes care of the tariff plans, creates and manages them, creates financial reports that are stored in documents, and helps them to find out how much they must pay for the services they have received during the billing period. The system may also be in VoIP or other services. [8]

\subsection{Midleware}

Midleware is a software that can be described as the SetTop box operating system. It switches the Set-top Box with the main distribution station. Using it we can get access to TV channels, video on demand, video recorder and all other features that are provided by the main distribution station in the Set Top Box. [8]

\subsection{IP Set-top Box}

Set-top Box is the end device used to receive TV, media, or other content from the main distribution station. The device is the endpoint of the network, located in the user's home. Its job is to decode the received service and send it to IPTV in the form of an image or sound, if it is a radio service. [8]

\section{IPTV architecture in IMS}

Figure 1 shows the IPTV architecture in the IMS network. We divide it into major parts:

- IMS core,

- media delivery and control features,

- transport functions,

- application server, - user device $[1,10,11]$ :

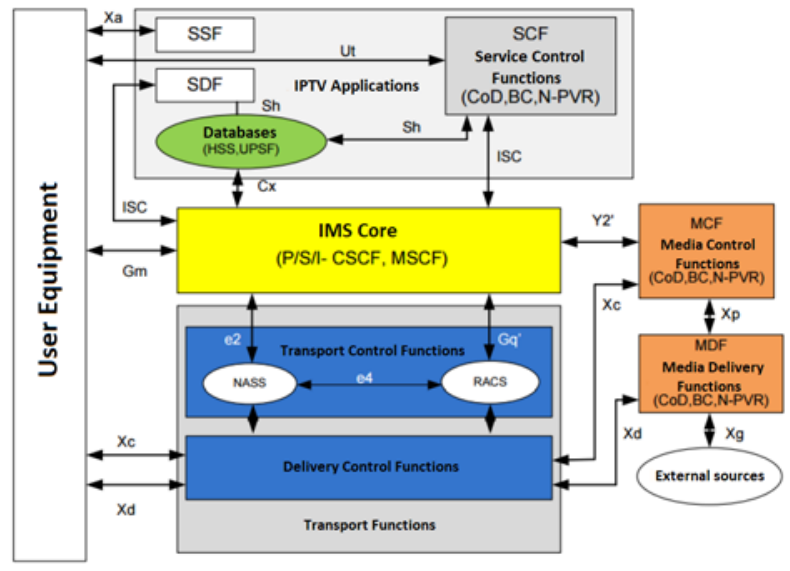

Fig. 1. IPTV architecture in IMS.

\subsection{IMS core}

Its most important task is signaling using the SIP protocol to manage connections in the IMS network using CSCF and HSS as previously described. The first point of communication between the IPTV customer and 
the IMS through the Gm reference point is the P-CSCF. It guarantees transmission of signaling over the network and allocates resources for communication.

Another point is S-CSCF, where customers are registered or authenticated. S-CSCF cooperates with HSS via the Cx reference point, HSS stores customers' data and provides them when needed. [12]

I-CSCF communicates with the S-CSCF and requests information from the HSS, which then selects and assigns the appropriate S-CSCF to the customer. $[10,13]$

\subsection{Media Control Functions}

Starajú sa o ochranu stiahnutého obsahu. Media Control Functions (MCF) are control functions, the main function of which is the monitoring and management of MDF (Media Delivery Functions). MDF creates reports about its status for MCF. MDF functions are responsible for delivering multimedia content to the customer, enabling the storage of media content if requested by the customer. They take care about the downloaded content. [10]

\subsection{Delivery and media control functions}

Transport functions work under the supervision of two RACS subsystems (Resource Admission Control Subsystem) and NASS (Network Attachment Subsystem).

The RACS is a resource management and input control subsystem. Used to perform access control, it is responsible for reserving the bandwidth for both multicast and unicast in the backbone networks. It also uses the Network Address Translation (NAT) protocol to transfer network addresses when the network passes from private to public. The RACS communicates with the IMS through the Gq reference point. NASS is a network connection subsystem. It is reminiscent of a DHCP server because, just like DHCP, NASS also assigns IP addresses. Using information provided by the HSS, it authorizes customer access to the network. With IMS core, the subsystem communicates via the e2 reference point and provides IMS signaling messages. NASS also communicates directly with the RACS through the e4 reference point and exchanges user profile information. [10]

\subsection{Application server}

The application server takes care of providing services such as IPTV. Every user is stored in the HSS and all information about what services the user has at his disposal. $[1,10]$

\subsection{User equipment}

By user device, each end device that delivers the service to a customer, whether it is a VoIP service or an IPTV, is meant. Specifically, in IPTV is the most commonly used like end device set-top box or personal computer.

\section{Design of an IPTV network architecture}

In our paper, we designed a network visible in the figure 2 , which has the role of receiving IPTV broadcasting. The core of the network is the DVB-T2 Evolveo Mars USB tuner that provides us to capture and subsequent distribution of DVB-T broadcasts. The core of the network is a virtual server running Linux Mint 18.1 Cinnamon, running on VirtualBox on a computer running Windows 7.

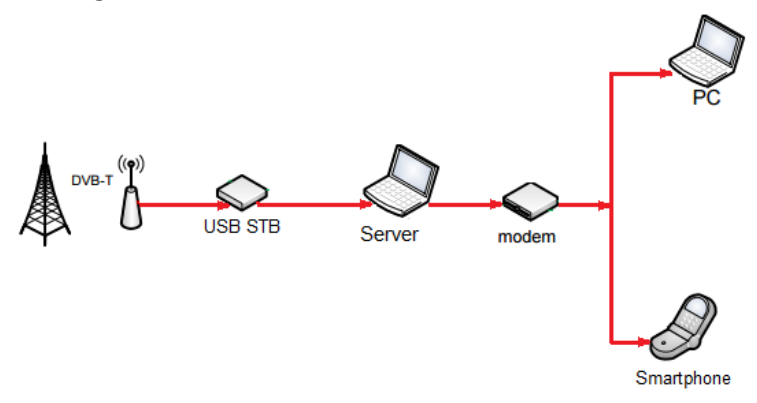

Fig. 2. The architecture of the proposed test network.

On the server, we installed the necessary driver that enabled us to communicate with the USB tuner. We needed to test the functionality of the device. The Wscan program is used to test the device. Figure 3 shows how $\mathrm{W}$-scan searches for individual frequencies. If there is a service on a given frequency, whether it is a TV or a radio station, W-scan will show the exact type of modulation used in the broadcast. In our case, W-scan services were found at $482,000 \mathrm{~Hz}$ and QAM 64 modulation was used. At frequencies where only time is available, no broadcast services were found.

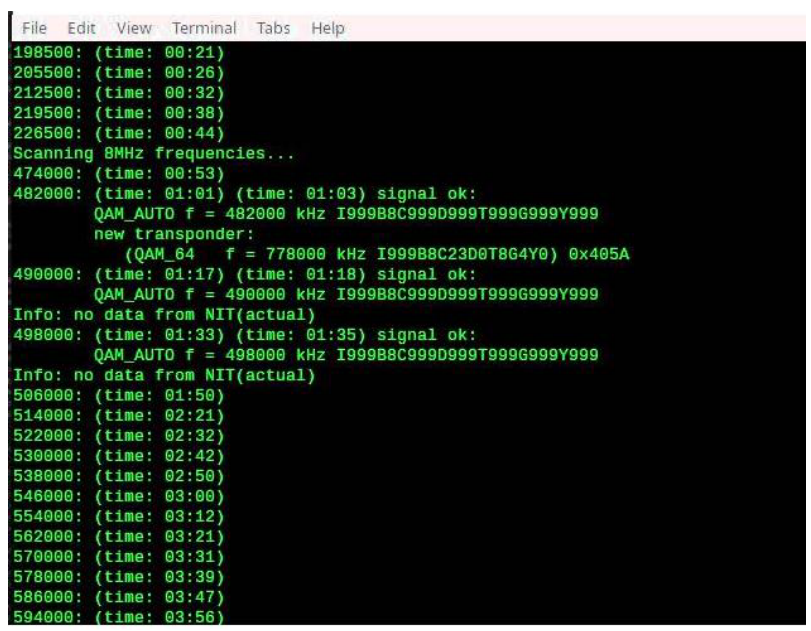

Fig. 3. W-scan multiplexes crawling.

Using the $\mathrm{W}$-scan program, we can set the country we are in, as well as which multiplexes we will search for the country. Multiplexes are in other words channels with predefined frequency. Each channel has a different frequency. For DVB-T, in Slovakia, it is possible to broadcast channels from $177.5 \mathrm{MHz}$ - channel 5 to 858 $\mathrm{MHz}$, representing channel 69.

In the Bratislava Region, it is possible to tune into 3 multiplexes, specifically on the $522 \mathrm{MHz}$ channel 27, 
offering STV1, STV2, STV1 HD, STV2 HD channels, on a $658 \mathrm{MHz}$ channel 44 with WAU, TV LUX, TV8 and channel 75 with a frequency of $754 \mathrm{MHz}$ with stations Infokanál, TV JOJ, JOJ Plus, Barrandov family and TA3.

Consequently, we needed to record the received signal. Because $\mathrm{W}$-scan does not show what services are available on the available channels, we needed to install TV-HeadEnd. TV-HeadEnd is a program that allows you to search for different types of broadcasts, such as satellite, cable, terrestrial, or IPTV. It also allows you to create users and manage your network. Each user can have limited access to services depending on which settings we allow users to use. To preview the functionality of the network, we have enabled all the services provided. With TV-HeadEnd, a local server with an IP address is created as the computer's address and 9981 port is accessed. Then, using the IP address and the accessed port, the user logs in with the name and password to the server. Figure 4 shows the program's configuration interface as well as the IP address and port in this case 10.14.0.253:9981.

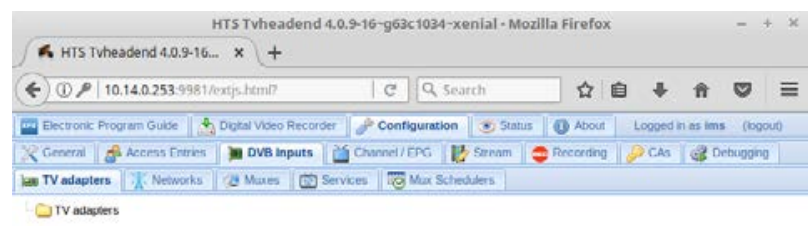

Fig. 4. TV-HeadEnd Environment.

It is also possible to see in figure 4 that we are in the configuration section which has its subsections. The first and probably the most important subsection is the subsection TV Adapters, which allows us to select the source of the incoming TV signal. We have chosen our USB tuner to receive terrestrial broadcasting as a signal source. Subsequently, we created a virtual network called IMS. It was needed to connect this network to the selected TV adapter. After connecting the adapter and the network, we have set multiplexes with the channels mentioned above in the network that will be monitored during the run. Figure 5 shows the multiplexes and the final state after their searching. We can note that multiplexes with a frequency of 522 and $778 \mathrm{MHz}$ are reported to fail. The reason is that the tuner did not have sufficient signal quality at $522 \mathrm{MHz}$ and $778 \mathrm{MHz}$ did not transmit any stations in the Bratislava region, but the frequency is functional in the Nitra region, where we tested the network for the first time with the intention of tuning regional television.

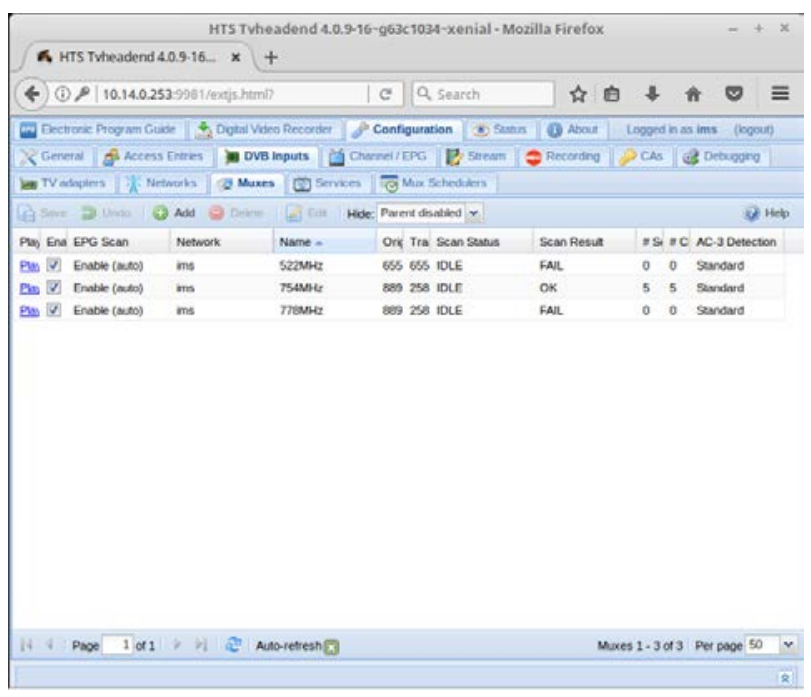

Fig. 5. The result of multiplexing.

After searching for all the multiplexes, we had $5 \mathrm{TV}$ stations - TV JOJ, JOJ Plus, Barrandov family, TA3 and Infokanál. The number of stations found depends on the quality of the received signal. This can be affected by the antenna's quality and type, weather, antenna position, and distance from the transmitter.

After searching, we needed to map all the stations. The mapping serves to assign the name and serial number to the TV stations you are looking for, as well as to create a hyperlink to the station. These hyperlinks we created and later were used to create a TV list that is accessed through our private network, for example through VLC media player. Each received TV station provides an EPG list as well as a teletext feature that gives us information about ongoing broadcasts, such as the year of production of the movie or the broadcast program, the direction information, the duration of the broadcast, the next broadcast program, etc. Unfortunately, the not available in VLC, but EPG is supported.

We can use almost every device with monitor and Internet access to monitor the stations. In our network, we tried to receive broadcast on a mobile device, on a smart TV and personal computer.

With open source VLC player, we were able to track mapped stations with every device connected to the private network.

Figure 6 shows how the created hypertext addresses appear in the $\mathrm{m} 3 \mathrm{u}$ list. 
\#EXTM3U

TINF : - 1, Barrand oy FAMILY

http://10.14.0.253:9981/stream/channelid/364531326?ticket= 3BF48EA7541835607FB472A622 92B818B1CE74AF \& profile=matrosk \#EXTINF: -1 , Infokanal

http://10.14.0.253:9981/stream/channelid/983038550?ticket= $140 \mathrm{~A} 25 \mathrm{EF} 43394 \mathrm{~A} 64565 \mathrm{D} 3 \mathrm{~F} 84 \mathrm{D} 9 \mathrm{AF} 85028 \mathrm{~F} 2 \mathrm{AC} 212 \& \mathrm{profile}=$ matroska \&profile=matroska

\#EXTINF: -1 , JOJ Plu

http://10.14.0.253:9981/stream/channelid/1224964305? ticket= 692548B7FAB4 675BA97D788B96928F789F705991\&profile=matroska \#EXTINF: -1, TA

http://10.14.0.253:9981/stream/channelid/1296410441?ticket= 5F 9AAFEA3B8AAAE5C668FDD2A23 9DA3D7C8869B3 \& profile=matroska \#EXTINF: -1 , TV JOJ

http://10,14.0.253:9981/stream/channelid/2042023597?ticket= 00730FDF3DE703572AE40A68FDEB064 9B0A292E2 \&profile=matroskal

Fig. 6. List of TV stations after mapping in hypertext format.

After running the list in the VLC media player, we found that even though we had the highest quality of the received signal, IP broadcasting had in some cases very low quality. This issue was mainly about HD stations, Jednotka HD and Dvojka HD, which we tested in the Nitra region when searching for regional stations. The whole problem is caused by the lack of bandwidth supported by TV-HeadEnd, so viewing in HD is almost impossible. For the comparison, figure 7 shows the SD transmission on the left side and the HD transmission on the right. While SD transmission is clean with minimal quality problems, HD transmission is sometimes just a green screen without sound.

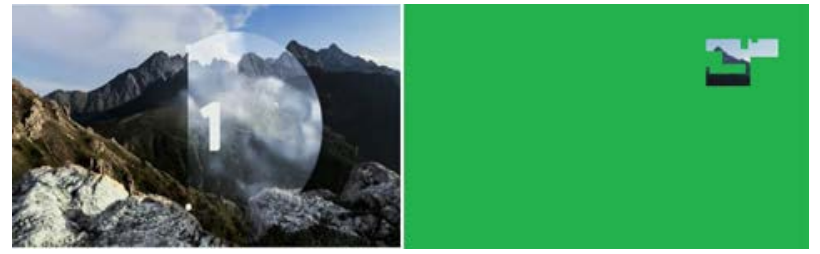

Fig. 7. Compare SD with HD.

Figure 7 also shows a rectangular image. The image shows an accurate view of what a large error occurs when many packets are lost, damaged, or not transmitted and discarded during transmission. The green area is the effort of the program to fill empty spaces that could not be delivered. However, as it is a lot, it seems earlier, as if the program tried to fill the green area with the received packets.

\section{Comparing quality of service}

Finally, we compare the quality of service provided by our network at the maximum load. As we saw in Figure 7, our network managed to send a high-quality signal when it was an SD transfer while it failed completely to send HD transmission. We tested on the network how it works under the burden of transmitting SD stations. Software D-ITG can simulate network load. It is needed to set the packet expedition interval exponentially, because it best simulates a crash overflow. We concealed the phenomenon of Bottleneck. It occurs with excessive network load during peak hours of operation, is limited by system capacity and is affected primarily by operating delays as well as hardware or software. [14, 15] Therefore, it is always necessary to consider and estimate how the network can be loaded at the peak of the network design and, accordingly, properly select switches and sufficiently high-performance hardware and high-quality software. The design will result in the full quality of the service, which is a key parameter in the provision of services, not just IPTV, but also others, such as VoIP, etc. The figure 8 and the figure 9 below show what defects occurred during the test overflow.

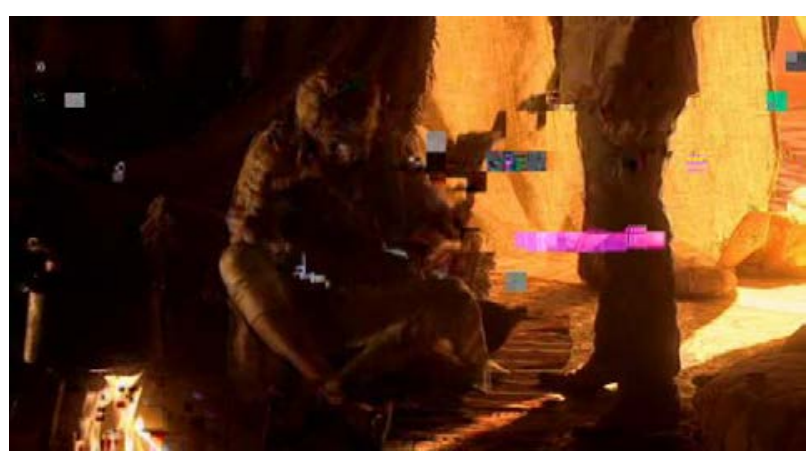

Fig. 8 Deprecated of transfer by packet loss.

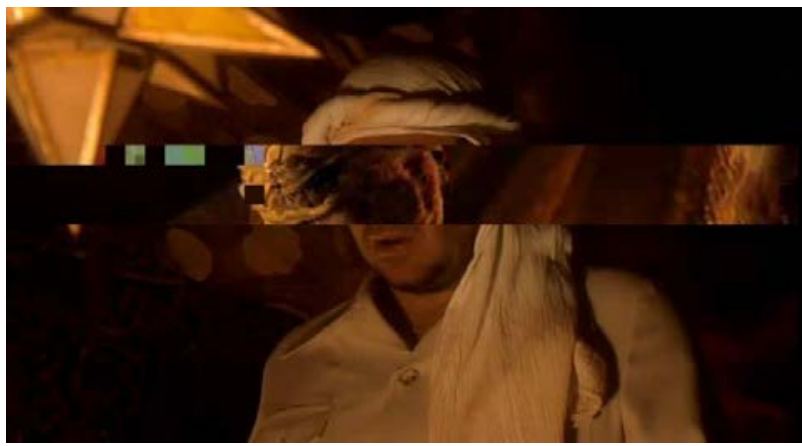

Fig. 9. Deprecation of transfer by packet loss and delays transmission.

By measuring, we have verified how the network load and delays significantly affect service quality. We have found that the most important step in designing a service in terms of quality is to ensure sufficient bandwidth for all users, as well as sufficient powerful software and hardware to take care of the distribution of the service.

\section{Conclusion}

To compare the quality provided by our network, we can use the network provided by one of the biggest Slovak operator. This service has plenty of bandwidth, high-quality TV source, high-quality hardware, and software designed to deliver IPTV and guaranteed service quality. It can deal with more connected users than our network. Just as our network, compared service supports the recording function, the EPG function, the 
teletext feature. The difference is that the recording function is a paid feature.

Our network could be improved by buying a more powerful signal receiver by purchasing a more powerful server that takes care of signal transfer and its distribution over the IP network, creating better quality software that can send data at a higher transmission rate. In the future, we assume hight probability of increasing number of divergent functions provided along IPTV. There is possibility to connect IPTV and smart television with smart household euuipment located in one private network. We see huge space to focuse on this field in our next research.

\section{Acknowledgement}

This article was created with the support of the Ministry of Education, Science, Research and Sport of the Slovak Republic within the KEGA agency project 007STU4/2016 Progressive educational methods in the field of telecommunications multiservice networks and VEGA agency project - 1/0462/17 Modeling of qualitative parameters in IMS networks.

\section{References}

1. D. Sivchenko, E. Mikoczy, V. Rakocevic, IMS based IPTV services - Architecture and Implementation, [online], Available at: http://www.staff.city.ac.uk/ veselin/publications/Siv chenko_ACM07.pdf

2. Z. Bosternak, R. Roka, Approach of the T-CONT allocation to increase the bandwidth in passive optical networks, In Radioengineering 26 (4), pp. 954-960, (2017)

3. IP Multimedia Subsystem (IMS), Functional architecture, [online]. Version ETSI ES 282007 2006, Available

at: http://www.etsi.org/deliver/etsi_es/282000_282099/ 282007/01.01.01_50/es_2 82007v010101m.pdf

4. IMS, IP Multimedia Subsystem Tutorial, [online], Available at: http://www.radioelectronics.com/info/telecommunications_networks/ ims-ip-multimedia-subsystem/tutorial-basics.php

5. F. Certik, R. Roka, Possibilities for Advanced Encoding Techniques at Signal Transmission in the Optical Transmission Medium, In Journal of Engineering, Vol. 2016, Article ID 2385372, 10 pp, DOI:10.1155/2016/2385372, (2016)

6. S. Klucik, J. Taraba, M. Orgon, D. Adamko, The Use of PLC Technology in Broadband Services Offered to Households, In International Journal of Information Technology and Computer Science (IJITCS). Vol. 4, No. 4, (2012)

7. A. Smakri, Andreas Strikos, A media agnostic peerto-peer architecture for IPTV distribution, KTH Information and Communication Technology, Stockholm, Sweden, COS/CCS 2008-11, (2008)
8. G. O'Driscoll, Next Generation IPTV Services and Technologies, JOHN WILEY \& SONS, INC., Publication, New Jersey, ISBN 978-0-470-16372-6, (2008)

9. P. Se Miikka, G. Mayer, H. Khartabil, A. Niemi, The IMS: IP Multimedia Concepts and Services, John Wiley \& Sons, Ltd. ISBN 0-470-01906-9, (2006)

10. Telecommunications and Internet converged Services and Protocols for Advanced Networking (TISPAN), [online], Available at: http://www.etsi.org/deliver/etsi_es/282000_282099/ 282001/02.00.00_50/es_282001v020000m.pdf

11. S. Klucik, E. Chromy, I. Baronak, Model to increase the number of output sates of a random variable using a histogram based PDF. In: Wireless Personal Communications, Vol. 85, Issue 1 (2015), page 137149, SPRINGER, New York, 2015, ISSN: 09296212, DOI 10.1007/s11277-015-2731-5.

12. M. Kellovsky, I. Baronak, M. Kavacky, E. Chromy, The Optimal Sizing of HSS Database in IMS, In Wireless Personal Communications, SPRINGER, New York, Vol. 96, Issue 4, pp. 5453-5466, ISSN: 0929-6212, DOI: 10.1007/s11277-016-3750-6, (2017)

13. F. Chamraz, I. Baronak, Impact of admission control methods to the traffic management. In: Advances in Electrical and Electronic Engineering, Vol., 13, Issue 4, 2015, pp. 280-288, ISSN: 1336-1376, 10.15598/aeee.v13i4.1437.

14. M. Halas, S. Klucik, Modelling the probability density function of IPTV traffic packet delay variation, In Advances in Electrical and Electronic Engineering, pp. 259-263. ISSN: 1336-1376, (2012)

15. J. Misurec, M. Orgon, Modeling of power line transfer of data for computer simulation, In International Journal of Communication Networks and Security, 3 (2), pp. 104-111, (2011) 